\title{
Organophosphate Esters in Children and Adolescents in Liuzhou City, China: Concentrations, Exposure Assessment and Predictors
}

\section{Meng Yu}

Tongji Medical College of Huazhong University of Science and Technology: Huazhong University of Science and Technology Tongji Medical College

\section{Xiang Li}

Tongji Medical College of Huazhong University of Science and Technology: Huazhong University of Science and Technology Tongji Medical College

\section{Bingqing Liu}

Women's Hospital School of Medicine Zhejiang University

\section{Yaping Li}

Tongji Medical College of Huazhong University of Science and Technology: Huazhong University of Science and Technology Tongji Medical College

\section{Ling Liu}

Tongji Medical College of Huazhong University of Science and Technology: Huazhong University of Science and Technology Tongji Medical College

\section{Limei Wang}

Tongji Medical College of Huazhong University of Science and Technology: Huazhong University of Science and Technology Tongji Medical College

\section{Lulu Song}

Tongji Medical College of Huazhong University of Science and Technology: Huazhong University of Science and Technology Tongji Medical College

\section{Youjie Wang}

Tongji Medical College of Huazhong University of Science and Technology: Huazhong University of Science and Technology Tongji Medical College

\section{Liqin Hu}

Wuhan Childrens Hospital: Wuhan Women and Children Medical Care Center

Surong Mei ( $\nabla$ surongmei@hust.edu.cn )

Huazhong University of Science and Technology Tongji Medical College

\section{Research Article}

Keywords: Organophosphate esters, urine, hand wipes, sociodemographic characteristics, dietary factors, children and adolescents

Posted Date: December 23rd, 2021

DOl: https://doi.org/10.21203/rs.3.rs-958515/v1

License: (c) (i) This work is licensed under a Creative Commons Attribution 4.0 International License. Read Full License 
Version of Record: A version of this preprint was published at Environmental Science and Pollution Research on January 31st, 2022. See the published version at https://doi.org/10.1007/s11356-021-18334-0. 


\section{Abstract}

Dermal contact with dust is commonly considered an important pathway of exposure to organophosphate esters (OPEs), but the importance of OPE uptake from diet is unclear. Herein, we used hand wipes to estimate OPE exposure from indoor dust and examined whether urinary OPE metabolite concentrations were influenced by sociodemographic characteristics, OPE amount in hand wipes, and dietary factors. OPEs were measured in urine and hand wipes from 6-18-year old children and adolescents $(n=929)$ in Liuzhou, China. Sociodemographic and dietary factors were obtained from questionnaire. Six OPE metabolites were detected in $>70 \%$ of the urine samples, and seven OPEs were detected in $>50 \%$ of the hand wipes. Estimated daily intakes (EDIs) were calculated using urinary OPE metabolites to investigate the total daily intake of OPEs, in which $0.36-10.1 \%$ of the total intake was attributed to the exposure from dermal absorption. In multivariate linear regression models, sex, age, and maternal education were significant predictors of urinary OPE metabolite concentrations. Urinary diphenyl phosphate (DPHP) is positively associated with its parent compounds 2-ethylhexyl-diphenyl phosphate (EHDPP) and triphenyl phosphate (TPHP) in hand wipes. High versus low vegetable intake was associated with a 23.7\% higher DPHP (95\% confidence interval (CI): 0.51\%, 52.1\%). Barreled water drinking was associated with a $30.4 \%$ (95\% Cl: 11.8\%, 52.0\%) increase in bis(1-chloro-2-propyl) 1-hydroxy-2-propyl phosphate (BCIPHIPP) compared to tap water drinking. Our results suggested the widespread exposure to OPEs in children and adolescents. In additional to dermal absorption, dietary intake may be an important exposure source of certain OPEs.

\section{Introduction}

Organophosphate esters (OPEs) are increasingly used as flame retardants and plasticizers in consumer and industrial products due to the phase-out of some polybrominated diphenyl ethers (PBDEs). It was reported that phosphorous and brominated flame retardants accounted for $11 \%$ and $23 \%$ of the total global consumption of flame retardants in 2008 , respectively. However, the percentage contribution of phosphorous flame retardants increased to $30 \%$, and brominated flame retardants attenuated to $20 \%$ in 2017 (Wang et al., 2020). Because OPEs are frequently physically mixed with polymers, they can easily released from treated products into the external environment, leading to a frequent detection of OPEs in air (Hou et al., 2021), sediments and waters (Ye et al., 2021), foodstuffs (Li et al., 2019a), and biota (Pantelaki and Voutsa, 2020). Concerns regarding these replacement OPEs have mounted, because toxicological studies suggested that some OPEs are considered endocrine disruptors (Liu et al., 2012), and carcinogenic- (Böckers et al., 2020), neurodevelopment- (Rock et al., 2020), and reproductive toxicants (Xu et al., 2017b).

Humans can be exposed to OPEs through different pathways, including dust ingestion and inhalation, dermal absorption, and dietary intake (Cequier et al., 2015; Liu et al., 2017). After being absorbed, OPEs could be rapidly metabolized (several hours to days) and excreted into the urine (Hou et al., 2016). Urinary OPE metabolite concentrations reflect integrated exposure from different pathways and have been extensively recognized as biomarkers of exposure to OPEs. A growing body of studies have estimated the total daily intake of OPEs through the monitoring of urinary OPE metabolite concentrations in the general population (Zhang et al., 2018b), children (Bastiaensen et al., 2021; Chen et al., 2018; Zhang et al., 2018a), and pregnant women (Bai et al., 2019; Chen et al., 2019). Additionally, the contamination levels of OPEs in indoor dust have been used to estimate nondietary exposure, which was considered one of the most important exposure pathways. To identify individual's exposure from indoor microenvironments, hand wipes could be an effective metric because it reflects the exposure from dermal contact with dust and other media (e.g., indoor consumer products) (Larsson et al., 2018). Similar dermal exposure estimates were obtained for several halogenated flame retardants (HFRs) when the exposure assessment was based on direct measurements from hand wipes or the indirect measurement from indoor dust (Tay et al., 2018). Human dermal absorption of OPEs could be estimated using the mass of OPEs in hand wipes, which has been reported in biomonitoring studies (Liu et al., 2017; Xu et al., 2016).

Although several studies have previously suggested that dermal contact with dust and inhalation are two more important pathways (He et al., 2018c; Wei et al., 2015; Xu et al., 2016), there is increasing concern regarding the occurrence of OPEs in foods, and the importance of diet as an exposure source of OPEs has been studied (Li et al., 2019a). Zhang et al. (2016) monitored the residue levels of OPEs in 75 commonly consumed foods in China and detected OPEs in $100 \%$ of the food samples. He and Wang (2018) collected 87 food samples from Southeast Queensland, Australia, and found that OPEs and their metabolites were both frequently detected in various foodstuffs. However, the associations between dietary factors and the body

Page $3 / 20$ 
burden of OPEs in humans are unclear. In a Norwegian adult cohort, Xu et al. (2017a) detected OPEs in duplicate diet samples $(n=61)$ and found that meat intake was the primary dietary exposure source of OPEs. A study conducted in eastern China showed that postpartum women $(n=50)$ who consumed more vegetables before pregnancy had a higher tris(2-chloroisopropyl) phosphate (TCIPP) level in placentas than those who consumed few vegetables (Ding et al., 2016). A study of toddlers aged 1518 months in the USA $(n=41)$, using dietary survey data, reported that meat and fish consumption may be linked with increased bis(1,3-dichloro-2-propyl) phosphate (BDCIPP) and diphenyl phosphate (DPHP) concentrations in urine (Thomas et al., 2017). Nevertheless, research using a larger sample size is warranted to verify the previous results.

Children and adolescents have more rapid nutrient absorption, metabolic and ventilation rates, and larger ratio of surface area to body weight as compared to adults (Van den Eede et al., 2015), thus, the body burden of OPEs in children and adolescents may be higher. The monitoring surveys in China suggested that children and adolescents are ubiquitously exposed to OPEs, especially chlorinated alkyl phosphates (Chen et al., 2018; Ding et al., 2019). Childhood and adolescence are in the critical periods of development, and developing organ systems are more susceptible to chemical exposure, including OPEs (Scheuplein et al., 2002). Considering increasing exposure to OPEs and their potential health risks, determining the factors that may impact OPE exposure in children and adolescents has been of growing concern. However, the related studies are very limited, especially in China (Bastiaensen et al., 2020; Bastiaensen et al., 2019; Bastiaensen et al., 2021; Phillips et al., 2018).

Therefore, we measured OPEs in urine and hand wipe samples from children and adolescents aged 6-18 years in Liuzhou, China. The purpose of this study was to determine 1) the exposure levels of OPEs in children and adolescents; 2) the contribution of dermal exposure dose to the total daily intake of OPEs; and 3) the associations between urinary OPE metabolites and OPE mass in hand wipes, as well as sociodemographic characteristics and dietary factors.

\section{Methods}

\subsection{Study design}

From February 2018 to May 2018, a total of 1388 children and adolescents (6-18 years old) were recruited from a crosssectional study in Liuzhou, China. The participants were selected from eight schools, including three primary schools, three middle schools, and two high schools. Of participants, 1194 persons provided urine samples and 1228 persons provided hand wipe samples, in which 1034 persons had paired urine-hand wipes. After exclusion of participants with missing data on dietary assessment, our final analysis consisted of 929 children and adolescents who provided urine and hand wipe samples, and had complete data on dietary assessment. The detailed information on the selection of the study participants was provided in Fig. S1.

Informed consent was provided by the participant's parents. Ethical approvals were obtained from Tongji Medical College, Huazhong University of Science and Technology.

\subsection{Dietary assessment}

Information on dietary factors was obtained using food frequency questionnaires, which is frequently used in large sampling campaigns. The questionnaire was provided to obtain the dietary patterns for the study participants, and they completed the questionnaire with their parents and were guided by trained researchers. In the questionnaire, seven frequently consumed food groups were administrated in the food items, including four foods of animal origin (meat, fish, eggs, and dairy products) and three foods of plant origin (vegetables, fruits, and beans). The consumption rate (per week) of the seven food groups is detailed in Table S1 and was categorized into tertiles for further analysis (Table 1). Additionally, the daily source of drinking water was recorded, and a four-category option was set in this item, including tap water, barrelled water, purified water, and others (e.g., well water). 
Table 1

Sociodemographic and dietary characteristics of children and adolescents.

\begin{tabular}{|c|c|}
\hline Variable & Mean \pm SD or $n(\%)$ \\
\hline Age (year) & $12.5 \pm 3.2$ \\
\hline \multicolumn{2}{|l|}{ Sex } \\
\hline Male & $431(46.4)$ \\
\hline Female & $498(53.6)$ \\
\hline \multicolumn{2}{|l|}{ Maternal education } \\
\hline Less than high school & $482(51.9)$ \\
\hline High school & $229(24.7)$ \\
\hline College or above & $218(23.4)$ \\
\hline \multicolumn{2}{|l|}{ Meat (times/week) } \\
\hline$\leq 2$ & $287(30.9)$ \\
\hline $3-5$ & $360(38.8)$ \\
\hline$\geq 6$ & $282(30.4)$ \\
\hline \multicolumn{2}{|l|}{ Fish (times/week) } \\
\hline 0 & $328(39.5)$ \\
\hline 1 & $331(35.6)$ \\
\hline$\geq 2$ & $270(29.1)$ \\
\hline \multicolumn{2}{|l|}{ Eggs (times/week) } \\
\hline$\leq 2$ & $270(29.1)$ \\
\hline 3 & $374(40.2)$ \\
\hline$\geq 4$ & $285(30.7)$ \\
\hline \multicolumn{2}{|c|}{ Dairy products (times/week) } \\
\hline$\leq 2$ & $333(35.8)$ \\
\hline $3-5$ & $301(32.4)$ \\
\hline$\geq 6$ & $295(31.8)$ \\
\hline \multicolumn{2}{|l|}{ Fruits (times/week) } \\
\hline$\leq 3$ & $378(40.7)$ \\
\hline $4-5$ & $259(27.9)$ \\
\hline$\geq 6$ & $292(31.4)$ \\
\hline \multicolumn{2}{|c|}{ Vegetables (times/week) } \\
\hline$\leq 4$ & $289(31.1)$ \\
\hline $5-6$ & $176(19.0)$ \\
\hline$\geq 7$ & $464(49.9)$ \\
\hline Beans (times/week) & \\
\hline
\end{tabular}

Page $5 / 20$ 


\begin{tabular}{|ll|}
\hline Variable & Mean \pm SD or $\mathbf{n}(\%)$ \\
\hline$\leq 1$ & $367(39.5)$ \\
\hline 2 & $233(25.1)$ \\
\hline$\geq 3$ & $329(35.4)$ \\
\hline Drinking water & \\
\hline Tap water & $586(63.1)$ \\
\hline Barreled water & $205(22.1)$ \\
\hline Purified water & $131(14.0)$ \\
\hline Others & $7(0.8)$ \\
\hline
\end{tabular}

\subsection{Sample collection}

During the school visit, a $5 \mathrm{~mL}$ polypropylene container marked with a unique identification number was given to each participant for spot urine collection. The collected urine samples were placed in carton and transferred to the laboratory.

The collection of hand wipes was performed as described in our previous study (Tao et al., 2018). Briefly, a sterile gauze pad $(6 \times 8 \mathrm{~cm})$ soaked in $5 \mathrm{~mL}$ of isopropanol was used to wipe the palm and back of each subject's hands. Subsequently, the collected hand wipe samples were enclosed in $50 \mathrm{~mL}$ polystyrene centrifuge tubes. Meanwhile, field blank samples were collected from all the schools.

\subsection{Sample analyses}

Prior to urine analysis, specific gravity (SG) was detected using refractometer (Atago PAL-10S, Tokyo, Japan), and urinary OPE metabolites were adjusted for dilution before statistical analyses. Eight urinary OPE metabolites, including tris(2-chloroethyl) phosphate (TCEP), 1-hydroxy-2-propyl bis(1-chloro-2-propyl) phosphate (BCIPHIPP), BDCIPP, dibutyl phosphate (DBP), DPHP, 4hydroxyphenyl-diphenyl phosphate (4-OH-DPHP), bis(2-butoxyethyl) phosphate (BBOEP), and di-ocresyl phosphate \& di-p-cresyl phosphate (DoCP \& DpCP) were measured according to our previous method (Hu et al., 2019). $0.2 \mathrm{ng}$ and $2 \mathrm{ng}$ standard solutions spiked in the pooled urine samples were used as quality control (QC) samples. The mean spiked recovery from QC samples was acceptable and ranged from $79 \%-119 \%$.

The hand wipes samples were analyzed for TCEP, TCIPP, tris(1,3-dichloro-2-propyl) phosphate (TDCIPP), triphenyl phosphate (TPHP), 2-ethylhexyl diphenyl phosphate (EHDPP), tris(2-butoxyethyl) phosphate (TBOEP), tricresyl phosphate (TCrP), and tris(2ethylhexyl) phosphate (TEHP). The OPE measurement in hand wipes was modified based on a previous study (Tao et al., 2018). $2 \mathrm{ng}$ and $20 \mathrm{ng}$ standard solutions spiked in sample matrices were used as QC samples. The average spiked recovery of OPEs in the QC samples exhibited acceptable accuracy, with a range from $82-108 \%$.

\subsection{Exposure assessment}

Estimated daily intakes (EDIs) were assessed using urinary OPE metabolite concentrations to determine the total daily intake of OPEs in children and adolescents. The calculation for EDIs (ng/kg bw/day) was described as:

$$
E D I=\frac{C_{\text {mopes }} \times V_{\text {urine }}}{F_{u e} \times b w} \times \frac{M W_{p}}{M W_{m}}
$$

where $\mathrm{C}_{\mathrm{mOPEs}}(\mathrm{ng} / \mathrm{mL})$ is the SG-corrected concentrations of individual OPE metabolites in case of this study; $\mathrm{V}_{\text {urine }}(\mathrm{mL} /$ day) is the reference value of daily urinary volume (Zhang et al., 2018b); $F_{U E}$ (unitless) is the urinary excreted molar fraction of metabolite to its corresponding parent compound (Van den Eede et al., 2013); bw (kg) is the body weight; $\mathrm{MW}_{\mathrm{p}}$ and $\mathrm{MW}_{\mathrm{m}}(\mathrm{g} / \mathrm{mol})$ 
are the molecular weights of the parent compound and its metabolite respectively. The parameters for estimating EDI were showed in Table S2-S3.

Dermal exposure dose (DED, ng/kg bw/day) of OPEs was estimated based on the amount of OPEs in hand wipes and was calculated as:

$$
D E D=\frac{C_{S} \times S A \times A F \times E D}{b w}
$$

where $\mathrm{C}_{\mathrm{S}}\left(\mathrm{ng} / \mathrm{m}^{2}\right)$ is individual OPE concentration on hand surface; SA $\left(\mathrm{m}^{2}\right)$ is the exposed surface area (USEPA, 2011); AF (unitless) is absorption factor adopted from human ex vivo skin experiments (Abou-Elwafa Abdallah et al., 2016); ED (h day ${ }^{-1}$ ) is the exposure time, which was assumed as $24 \mathrm{~h}$; and bw $(\mathrm{kg})$ is the body weight. The parameters of SA among different age group and AF of individual OPEs were showed in Table S4-S5.

\subsection{Statistical analyses}

Extracted samples with OPE concentrations below the LOD were substituted with LOD $/ \sqrt{2}$. Compounds with a detection frequency $>50 \%$ were included in all statistical analyses, whereas we did not analyze TEHP in hand wipes due to the greatly varied concentrations of TEHP in the QC samples. The concentrations of urinary OPE metabolites were natural log-transformed due to the lack of normally distributed. Spearman correlation coefficients $\left(r_{s}\right)$ were used to determine the associations between OPE metabolites in urine and OPEs in hand wipes.

Multivariate linear regression model was applied to investigate the potential predictors of urinary OPE metabolite concentrations. The following variables were included in the models: subject's sex and age (categorical variable), maternal education, the source of drinking water, the consumption frequency (per week) of seven food groups (meat, fish, eggs, dairy products, fruits, vegetables, and beans), and the concentrations of OPEs in hand wipes. OPE metabolites in urine and their corresponding parent compound in hand wipes were simultaneously entered into multivariable models, as described in Fig S2. Age was categorized as 6-11 years, 12-15 years, or 16-18 years. Maternal education was categorized as less than high school, high school, or college or above. The hand wipe levels were categorized into quartiles, except for TCEP and TBOEP. TCEP and TBOEP were categorized into tertiles and dichotomy, respectively, due to their low detection frequencies. Additionally, we tested multicollinearity using the variance inflation factor (VIF), with all VIFs $<5$. To interpret the regression models containing In-transformed outcome variables, the regression coefficient $(\beta)$ and $95 \%$ confidence interval $(\mathrm{Cl})$ was converted to percent change (\%change) and $95 \% \mathrm{Cl}$ in urinary OPE metabolite concentrations.

All analyses were carried out using STATA 12.0 (StataCorp, College Station, TX, USA). A two-sided $P<0.05$ was considered significant.

\section{Results And Discussion}

\subsection{Study population}

Of participants, $46.4 \%$ of children and adolescents were male and $53.6 \%$ were female, with a mean age of $12.5 \pm 3.2$ years old (Table 1). A total of $51.9 \%$ of mothers attained less than a high school degree, $24.7 \%$ attained equal to a high school degree, and $23.4 \%$ participated in college. The majority of food groups was generally categorized into tertiles, except for vegetable intake. A total of $49.9 \%$ of the participants were divided into the third tertile due to their high proportion of seven meals per week for vegetable consumption. The source of drinking water was mainly tap water $(63.1 \%)$, followed by barreled water $(22.1 \%)$, purified water (14.0\%), and others (0.8\%).

\subsection{Concentrations of urinary OPE metabolites}

Table 2 displayed the concentration distributions of urinary OPE metabolites in children and adolescents. Six OPE metabolites (TCEP, BCIPHIPP, BDCIPP, DPHP, BBOEP, and DoCP \& DpCP) were detected in $>70 \%$ of the urine samples, suggesting widespread 
exposure to OPEs for the study participants. BCIPHIPP, TCEP, DPHP, and BDCIPP were the primary four metabolites in the urine samples, with SG-corrected median concentrations of $0.37 \mathrm{ng} / \mathrm{mL}, 0.27 \mathrm{ng} / \mathrm{mL}, 0.22 \mathrm{ng} / \mathrm{mL}$, and $0.19 \mathrm{ng} / \mathrm{mL}$, respectively.

Table 2

Descriptive statistics for OPEs and their urinary metabolites.

\begin{tabular}{|c|c|c|c|c|c|c|c|c|c|}
\hline \multirow[t]{2}{*}{ Analytes } & \multirow[t]{2}{*}{ Parent compound } & \multirow[t]{2}{*}{$>$ LOD $(\%)$} & \multirow[t]{2}{*}{ LOD } & \multirow[t]{2}{*}{ GM } & \multicolumn{4}{|c|}{ Percentile } & \multirow[t]{2}{*}{ Max } \\
\hline & & & & & 25th & 50th & 75th & 95th & \\
\hline \multicolumn{10}{|c|}{ SG-corrected urine (ng/mL) } \\
\hline TCEP & TCEP & 79.3 & 0.092 & 0.28 & 0.13 & 0.27 & 0.55 & 1.60 & 11.0 \\
\hline BCIPHIPP & TCIPP & 85.7 & 0.097 & 0.35 & 0.20 & 0.37 & 0.63 & 1.79 & 28.8 \\
\hline BDCIPP & TDCIPP & 78.1 & 0.064 & 0.18 & 0.07 & 0.19 & 0.41 & 1.04 & 6.06 \\
\hline DBP & TBP & 25.2 & 0.058 & 0.07 & $<\mathrm{LOD}$ & $<L O D$ & 0.08 & 1.08 & 7.70 \\
\hline 4-OH-DPHP & TPHP & 42.0 & 0.043 & 0.08 & $<$ LOD & $<L O D$ & 0.24 & 1.95 & 11.2 \\
\hline DPHP & TPHP/EHDPP & 91.7 & 0.036 & 0.24 & 0.10 & 0.22 & 0.49 & 2.03 & 25.5 \\
\hline BBOEP & TBOEP & 89.6 & 0.012 & 0.12 & 0.03 & 0.16 & 0.37 & 1.07 & 7.43 \\
\hline DoCP \& DpCP & $\mathrm{TCrP}$ & 70.7 & 0.032 & 0.06 & 0.03 & 0.05 & 0.10 & 0.29 & 1.89 \\
\hline \multicolumn{10}{|c|}{ Handwipes (ng/wipe) } \\
\hline TCEP & & 65.0 & 0.66 & 1.04 & 0.47 & 0.90 & 1.72 & 4.99 & 27.4 \\
\hline TCIPP & & 87.1 & 2.50 & 9.58 & 4.63 & 9.49 & 18.1 & 59.4 & 847 \\
\hline TDCIPP & & 81.1 & 0.80 & 2.09 & 1.01 & 2.02 & 3.85 & 13.2 & 64.3 \\
\hline TPHP & & 89.9 & 1.00 & 4.08 & 2.09 & 3.76 & 6.86 & 30.5 & 378 \\
\hline EHDPP & & 98.6 & 0.67 & 10.1 & 5.57 & 10.9 & 20.2 & 56.5 & 222 \\
\hline TBOEP & & 53.0 & 0.31 & 0.39 & $<L O D$ & 0.32 & 0.55 & 1.59 & 448 \\
\hline $\mathrm{TCrP}$ & & 93.0 & 0.30 & 0.80 & 0.48 & 0.78 & 1.26 & 3.20 & 80.2 \\
\hline
\end{tabular}

Median concentrations of urinary OPE metabolites in several studies were compared in Table S6. BCIPHIPP, BDCIPP, and DPHP are commonly analyzed urinary biomarkers of exposure to OPEs and are frequently detected in urine samples around the world. Compared to the concentrations in children or adolescents from the USA (Hoffman et al., 2018; Ospina et al., 2018), Belgium (Bastiaensen et al., 2021), Norway (Cequier et al., 2015), and Australia (He et al., 2018a; He et al., 2018b), these urinary metabolite concentrations in our study were relatively lower, but comparable to the results from other studies in China (Chen et al., 2018; Zhang et al., 2018a; Zhang et al., 2018b). BBOEP was detected in 90\% of the urine samples. The concentration of BBOEP in our participants was higher than that in children from central and southern China (Chen et al., 2018; Zhang et al., 2018a), comparable to that in adolescents from eastern China (Ding et al., 2019), but lower than the levels in children from Australia (He et al., 2018b). A larger amount of flame retardant usage or widespread exposure of OPEs may partly explain a higher urinary OPE metabolite concentrations in children from the developed countries. It was reported that the total OPE consumption in Europe and the USA was much higher than that in China for the same duration (Tao et al., 2021). The contamination levels of OPEs in indoor dust and air (Hou et al., 2021) and foods (Li et al., 2019a) are more pronounced in the developed countries. TCEP, as the parent compound of OPEs, could be detected in $80.5 \%$ of our participants. The concentration of urinary TCEP was comparable to that in adolescents from eastern China (Ding et al., 2019), but higher than that in children from Japan (Bastiaensen et al., 2019), Australia (He et al., 2018b), and Hong Kong, China (Li et al., 2019b). TCEP was found to be the most abundant OPEs in suspended particulate matters and gaseous phases (Ding et al., 2019; Mäkinen et al., 2009), and 
inhalation of TCEP was considered the greatest contributor of daily intake for TCEP (Xu et al., 2017a). Due to limited data available from multiple environmental compartments, whether a higher body burden of TCEP in children and adolescents of Liuzhou city is attributed to a higher contamination of TCEP in indoor air remains to be confirmed.

\subsection{Concentrations of OPEs in hand wipes}

The concentration distributions of OPEs in hand wipes from children and adolescents were shown in Table 2. Seven OPEs were detected in $>50 \%$ of the hand wipe samples. EHDPP and TCIPP were detected at the highest median concentrations of 10.9 $\mathrm{ng} /$ wipe and $9.49 \mathrm{ng} /$ wipe, respectively.

Concentrations of OPEs in hand wipes from various studies were summarized in Table S7. Two aryl phosphate compounds (TPHP and EHDPP) were ubiquitously detected in the general population. The median concentrations of TPHP and EHDPP in the present study were similar to those in a study of adults and children aged 1-5 years from South China (Tan et al., 2018), but slightly lower than the results reported from children aged 3-6 years in the USA (Hammel et al., 2020; Phillips et al., 2018). The detection frequency of TCrP in hand wipes is heterogeneous among different studies, but the median concentration of TCrP in our participants was comparable to that in toddlers or adults (Sugeng et al., 2017; Tao et al., 2018). Chlorinated alkyl phosphates (TCEP, TCIPP, and TDCIPP) were also frequently detected, with TCIPP having the highest median concentration of $9.49 \mathrm{ng} /$ wipe among this subclass of compounds. This finding was different from other studies reporting TDCIPP as the most abundant in chlorinated alkyl phosphates, suggesting differential exposure in different subpopulations (Phillips et al., 2018), or a possible regional difference between domestic and foreign exposure (Stapleton et al., 2014; Tan et al., 2018). OPEs were detected in almost $100 \%$ of the hand wipe samples in our study, while the concentrations of OPEs, particularly TBOEP, were significantly lower than those in studies of children from other countries. TBOEP was 3-4 orders of magnitude lower than that reported from the USA (Phillips et al., 2018), Sweden (Larsson et al., 2018), and Norway (Xu et al., 2016), probably because ceramic flooring, not flooring treated with polish or wax is commonly used in schools in China (Zhao et al., 2020).

\subsection{Exposure assessment}

To investigate the total daily intake of OPEs, EDIs of individual OPEs were calculated and displayed in Table 3. Due to the lack of data on toxicokinetics of OPEs, only EDI $\mathrm{TCIPP}_{\mathrm{T}} \mathrm{EDI}_{\mathrm{TDCIPP}}, \mathrm{EDI}_{\mathrm{TPHP}}$, and $\mathrm{EDI}_{\mathrm{TBOEP}}$ could be estimated in the present study. We only discussed the EDI values estimated based on Fue-S9-fraction, because Fue values from S9 fraction experiment contains both phase I and phase II metabolism (Van den Eede et al., 2013). The median EDIs of TCIPP and TPHP in our study were much lower than those reported in 0-5-year old children from central China (TCIPP: $94.9 \mathrm{ng} / \mathrm{kg}$ bw/day; TPHP: $267 \mathrm{ng} / \mathrm{kg}$ bw/day) (Zhang et al., 2018a) and 14-15-year old adolescents from Belgium (TCIPP: 57.4 ng/kg bw/day; TPHP: $197.7 \mathrm{ng} / \mathrm{kg}$ bw/day) (Bastiaensen et al., 2021), but comparable to the levels in 6-14-year old children from South China (TCIPP: $22.6 \mathrm{ng} / \mathrm{kg}$ bw/day; TPHP: 33.1 $\mathrm{ng} / \mathrm{kg}$ bw/day) (Chen et al., 2018). For TDCIPP, the median EDI was similar to that in several studies of general population (Bastiaensen et al., 2021; Chen et al., 2018; Zhang et al., 2018a; Zhang et al., 2018b). The EDI of TBOEP were heterogeneous in different studies, but generally within the same range. Compared to the reference dose (RfD) made for human exposure to TCIPP (10000 ng/kg bw/day), TDCIPP (20000 ng/kg bw/day), TPHP (20000 ng/kg bw/day), and TBOEP (15000 ng/kg bw/day) (Poma et al., 2019), the high exposure levels (95th percentile EDIs) of the study participants were $2-3$ orders of magnitude lower. Considering that RfD values were obtained based on toxicological data, the EDIs of individual OPEs in the present study could be over- or underestimated. 
Table 3

Estimated daily intakes ( $\mathrm{ng} / \mathrm{kg}$ bw/day) of OPEs.

\begin{tabular}{|c|c|c|c|c|c|c|c|c|c|c|c|c|}
\hline \multirow[t]{3}{*}{ Compound } & \multirow{3}{*}{$\begin{array}{l}\text { RfD a } \\
\text { (ng/kg } \\
\text { bw/day) }\end{array}$} & \multirow{2}{*}{\multicolumn{3}{|c|}{ Dermal exposure }} & \multicolumn{6}{|c|}{ Total daily exposure } & \multicolumn{2}{|c|}{ Proportion (\%) } \\
\hline & & & & & \multicolumn{3}{|l|}{ s9 } & \multicolumn{3}{|l|}{ HLM } & \multirow{2}{*}{$\begin{array}{l}\text { S9 } \\
\text { Median }\end{array}$} & \multirow{2}{*}{$\begin{array}{l}\text { HLM } \\
\text { Median }\end{array}$} \\
\hline & & Median & 5th & 95th & Median & 5th & 95th & Median & 5th & 95th & & \\
\hline TCEP & & 0.31 & 0.10 & 1.83 & & & & & & & & \\
\hline TCIPP & 10000 & 2.63 & 0.40 & 22.9 & 27.6 & 5.34 & 140 & 23.4 & 4.53 & 119 & 10.1 & 11.9 \\
\hline TDCIPP & 20000 & 0.32 & 0.06 & 2.36 & 7.70 & 1.20 & 45.8 & 11.4 & 1.78 & 67.7 & 4.69 & 3.17 \\
\hline TPHP & 20000 & 0.98 & 0.16 & 10.3 & 31.7 & 4.50 & 340 & 14.7 & 2.09 & 158 & 3.25 & 7.02 \\
\hline TBOEP & 15000 & 0.09 & 0.27 & 17.2 & 28.4 & 1.57 & 191 & 5.61 & 0.31 & 37.8 & 0.36 & 1.84 \\
\hline EHDPP & & 3.10 & 0.04 & 0.45 & & & & & & & & \\
\hline TCrP & & 0.24 & 0.06 & 1.22 & & & & & & & & \\
\hline$\Sigma_{7} \mathrm{OPES}$ & & 10.7 & 2.32 & 49.7 & & & & & & & & \\
\hline \multicolumn{13}{|c|}{ Note: RfD, reference dose; HLM, human liver microsomes; S9, S9 fraction. } \\
\hline
\end{tabular}

To investigate the contribution of dermal exposure to OPEs, the OPE intake via skin wipes and its proportion to total daily intake were estimated (Table 3). The median DED of $\Sigma_{7}$ OPEs was 10.7 (5.56-19.3) $\mathrm{ng} / \mathrm{kg}$ bw/day, in which TCIPP and EHDPP exposure via skin wipes were predominant contributors. The average proportion of OPE exposure via skin wipes ranged from $0.36 \%$ (TBOEP) to $10.1 \%$ (TCIPP) as total daily intake was estimated based on FUE-S9-fraction, suggesting a significant contribution of dermal absorption. In the present study, daily dermal exposure to OPEs of the study participants were higher than those estimated via hand wipes or house dust for adults (Cao et al., 2019; Huang et al., 2020; Liu et al., 2017; Xu et al., 2016), but lower than those estimated via house dust or air for toddlers (Huang et al., 2020; Liu et al., 2017). Different from other studies, we only considered the exposed surface area of hands and head as recommended by Pan et al. (2020). OPE uptake from dermal absorption could be higher if more skin area or whole body is included. Additionally, we adopted the absorption factors of TCEP (28\%), TCIPP (25\%), and TDCIPP (13\%) from human ex vivo skin experiments to investigate the daily dermal exposure to OPEs, and utilized the average absorption rate (22\%) of TCEP, TCIPP, and TDCIPP to estimate other OPEs (including TPHP, EHDPP, TBOEP, and TCrP).

\subsection{Correlation between urine and hand wipes}

We found that the TPHP and EHDPP levels in hand wipes were significantly correlated with their metabolite DPHP in urine $\left(r_{s}=\right.$ $0.068-0.142, P<0.05)$, whereas no significant correlations were observed between other OPEs in hand wipes and the corresponding urinary metabolites (Table 4). Hand wipes are theoretically a good integrated indicator of internal exposure, as they measured hand-to-mouth contact with dust and other exposure media (e.g., indoor consumer products), and may provide information on an individual's activity pattern. Several studies have reported strong correlations between the TCIPP and TDCIPP amount in hand wipes and their urinary metabolites of BCIPHIPP and BDCIPP, respectively (Phillips et al., 2018; Tao et al., 2018), whereas our study did not confirm these previous findings, possibly because the levels of OPEs on hands could be affected by individual's hand washing frequency and engaged activity before sampling (Larsson et al., 2018; Liu et al., 2017). However, hand washing frequency in our study was not related to the OPE concentrations in hand wipes (data not shown). Given that children and adolescents have a frequent contact with cell phone, the activity of cell phone touching was studied, as cell phones may act as a potential source of OPEs (Yang et al., 2019). There was no significant association between cell phone touching and the quantities of OPEs in hand wipes (data not shown). 
Table 4

Spearman correlation coefficients for OPE and OPE metabolites levels measured in

paired hand wipes and SG-corrected urine with $>50 \%$ detection ${ }^{a}$.

\begin{tabular}{|lllllll|}
\hline Compound & TCEP & BCIPHIPP & BDCPP & DPHP & BBOEP & DoCP \& DpCP \\
\hline TCEP & 0.034 & 0.016 & $\mathbf{0 . 0 7 3 ^ { * }}$ & 0.058 & $-0.072^{*}$ & 0.011 \\
\hline TCIPP & $-0.069 *$ & -0.048 & 0.022 & 0.042 & -0.003 & 0.013 \\
\hline TDCIPP & -0.007 & -0.059 & 0.016 & 0.018 & $-0.144^{*}$ & 0.002 \\
\hline TPHP & 0.033 & 0.047 & $\mathbf{0 . 0 9 7 ^ { * }}$ & $\mathbf{0 . 0 6 8 ^ { * }}$ & -0.010 & 0.043 \\
\hline EHDPP & -0.045 & -0.029 & $\mathbf{0 . 1 0 5 ^ { * }}$ & $\mathbf{0 . 1 4 2 ^ { * }}$ & 0.024 & 0.024 \\
\hline TBOEP & -0.030 & -0.039 & -0.010 & -0.004 & -0.016 & $-0.054^{*}$ \\
\hline TCrP & -0.048 & 0.035 & 0.035 & $\mathbf{0 . 0 6 2}$ & $\mathbf{0 . 0 8 7 ^ { * }}$ & 0.001 \\
\hline Note:
\end{tabular}

In the present study, OPE intake via dermal absorption could partially explain the body burden of OPEs. To determine the potential predictors for urinary OPE metabolite concentrations, factors (sociodemographic variables, dietary patterns, and OPE levels in hand wipes) that may impact OPE exposure were all included in multivariable linear regression models (Table 5). After adjusting for sex, age, maternal education, and dietary patterns, a strong correlation continued to be found for EHDPP in the highest quartile in hand wipes and urinary DPHP $(P<0.001)$. Although the association between TPHP and DPHP was no longer significant, there was a suggestive dose response relationship between TPHP and DPHP (Fig. 1). Similarly, the levels of other OPEs in hand wipes were not related to their corresponding urinary metabolites in the multivariable models. These results implied that hand wipes could be an effective exposure metric for TPHP and EHDPP, whereas the exposure of other OPEs may be linked to additional predictors. Furthermore, we discussed the associations of sociodemographic and dietary variables with urinary OPE levels after adjusted for other variables in Table 5. 
Table 5

Results of regression analyses for predicting urinary OPE metabolites ${ }^{a}$.

\begin{tabular}{|c|c|c|}
\hline OPE metabolites & Variables & \%change $(95 \% \mathrm{Cl})$ \\
\hline \multirow[t]{12}{*}{ TCEP } & Age & \\
\hline & 6-11 years (Ref) & \\
\hline & $12-15$ years & $97.6(68.5,132)$ * \\
\hline & $16-18$ years & $34.4(13.5,59.2)$ * \\
\hline & Sex & \\
\hline & Male (Ref) & \\
\hline & Female & $-14.3(-24.9,-2.31)$ * \\
\hline & Drinking water & \\
\hline & Tap water (Ref) & \\
\hline & Barreled water & $2.39(-13.2,20.7)$ \\
\hline & Purified water & $-8.67(-24.8,10.8)$ \\
\hline & Others & $-58.2(-80.3,-10.9)$ * \\
\hline \multirow[t]{9}{*}{ BCIPHIPP } & Age & \\
\hline & $6-11$ years (Ref) & \\
\hline & $12-15$ years & $41.7(22.1,64.4)$ * \\
\hline & $16-18$ years & $18.0(0.86,38.0)$ * \\
\hline & Drinking water & \\
\hline & Tap water (Ref) & $30.4(11.8,52.0)$ * \\
\hline & Barreled water & $16.7(-2.51,39.8)$ \\
\hline & Purified water & $-22.5(-61.7,57.0)$ \\
\hline & Others & \\
\hline \multirow[t]{8}{*}{ BDCIPP } & Age & \\
\hline & 6-11 years (Ref) & \\
\hline & $12-15$ years & $-4.24(-19.0,13.2)$ \\
\hline & $16-18$ years & $-48.4(-56.6,-38.5)$ * \\
\hline & Beans (times/week) & \\
\hline & $\leq 1$ (Reference) & \\
\hline & 2 & $2.11(-15.0,22.6)$ \\
\hline & $\geq 3$ & $-19.7(-32.4,-4.61)$ * \\
\hline \multirow[t]{3}{*}{ DPHP } & Age & \\
\hline & 6-11 years (Ref) & \\
\hline & $12-15$ years & $5.96(-12.4,28.2)$ \\
\hline
\end{tabular}

Note: ${ }^{\text {a }}$ Significant level are marked with bold text and asterisks $\left({ }^{*} P<0.05\right)$. 


\begin{tabular}{|c|c|c|}
\hline \multirow[t]{10}{*}{ OPE metabolites } & Variables & \%change (95\% Cl) \\
\hline & $16-18$ years & $-42.1(-52.5,-29.4)$ * \\
\hline & Meat (times/week) & \\
\hline & $\leq 2$ (Ref) & \\
\hline & $3-5$ & $-18.9(-33.3,-1.43)$ * \\
\hline & $\leq 6$ & $-9.52(-26.9,12.0)$ \\
\hline & Vegetables (times/week) & \\
\hline & $\leq 4$ (Ref) & \\
\hline & 2 & $13.3(-10.3,43.2)$ \\
\hline & $\geq 3$ & $23.7(0.51,52.1)$ * \\
\hline \multirow[t]{16}{*}{ BBOEP } & Age & \\
\hline & 6-11 years (Ref) & \\
\hline & $12-15$ years & $-21.6(-38.9,0.65)$ \\
\hline & $16-18$ years & $-6.74(-28.1,21.0)$ \\
\hline & Sex & \\
\hline & Male (Ref) & \\
\hline & Female & $22.3(0.75,48.5)$ * \\
\hline & Maternal education & \\
\hline & Less than high school (Ref) & \\
\hline & High school & $22.2(-4.40,56.1)$ \\
\hline & College or above & $50.9(17.5,93.6)$ * \\
\hline & Drinking water & \\
\hline & Tap water (Ref) & \\
\hline & Barreled water & $5.11(-17.6,34.1)$ \\
\hline & Purified water & $-27.3(-45.3,-3.46)$ * \\
\hline & Others & $33.1(-56.4,306)$ \\
\hline
\end{tabular}

Note: ${ }^{a}$ Significant level are marked with bold text and asterisks $(* P<0.05)$.

\subsection{Urinary OPE metabolites and sociodemographic characteristics}

It was not surprising that the urinary BDCIPP, DPHP, and BBOEP concentrations were inversely associated with age (categorical variable), because children have rapid metabolic and ventilation rates and large ratio of surface area to body weight, in addition to frequent hand-to-mouth activity (Miller et al., 2002; Tulve et al., 2002). Nevertheless, the levels of urinary TCEP and BCIPHIPP in the study participants aged 12-15 years were highest, followed by adolescents aged 16-18 years, and children aged 6-11 years were lowest. Contradictory to previous studies (Bastiaensen et al., 2019; He et al., 2018b; Van den Eede et al., 2015), we observed positive assocations of urinary TCEP and BCIPHIPP concentrations with age. To our knowledge, we did not observe a determinant activity pattern in which senior students had an increased potential for exposure to OPEs compared to schoolchildren, while a recent study found that urinary TCEP and BCIPHIPP levels were high in adolescents aged 12-15 years in eastern China (Ding et al., 2019). The concentration of urinary TCEP were high in males, while urinary BBOEP was high in 
females, indicating differences in metabolism and exposure patterns between genders (Hoffman et al., 2015). Maternal education level was a proxy for socioeconomic status that was positively associated with the urinary BBOEP in our participants. A potential explanation of this result is that newer furniture and electronics entered into these highly educated mothers' households, which led to the transition of indoor exposure patterns from PBDEs to OPEs (Percy et al., 2020).

\subsection{Urinary OPE metabolites and dietary patterns}

High vegetable intake in the past week ( $\geq 7$ times) was associated with a $23.7 \%$ higher DPHP concentration in urine compared to the lowest vegetable intake ( $\leq 4$ times) $[\%$ change $=23.7 ; 95 \%$ confidence interval $(\mathrm{Cl}): 0.51,52.1]$. Medium meat intake $(3-5$ times) was associated with a $18.9 \%$ lower DPHP concentration, although there was lack of statistical significance for the highest meat intake category ( $\geq 6$ times). In comparison with frequently consumed tap water, barreled water was associated with a $30.4 \%$ increased BCIPHIPP concentration in urine, purified water drinking was related to a $27.3 \%$ lower BBOEP concentration, and other drinking water was associated with a $58.2 \%$ lower TCEP concentration.

High vegetable intake was suspected to be an important factor that influence the concentrations of urinary OPE metabolites, which was first reported in children and adolescents. Zhang et al. (2016) detected 75 commonly consumed foods sampled from 4 representative areas in China and found that vegetables were severely contaminated by TCEP and TEHP. TPHP and EHDPP were not abundant OPEs in vegetables, thus, we could not conclude that the higher DPHP concentration in urine was attributed to the high contamination of its parent compounds in vegetables. However, a study in Australia reported the ubiquitous occurrence of DPHP in vegetables. The contamination level of DPHP (median: $0.99 \mathrm{ng} / \mathrm{g}$ wet weight) in vegetables was obviously higher than its parent compound TPHP (He and Wang, 2018). Considering the ubiquitously co-existence of organophosphate di-esters (di-OPEs) and tri-esters (tri-OPEs) in various environmental compartments (Björnsdotter et al., 2018; Hou et al., 2017; Tan et al., 2019), the direct intake of di-OPEs from foodstuffs cannot be ignored.

Negative associations between food consumption and urinary metabolite concentrations are challenging to interpret and are rarely reported. Eggs, fish, and meat which are categorized as animal-based foods, had the lowest contamination concentrations of OPEs compared to other food groups (Li et al., 2019a). The very low OPE concentrations in the animal-based foods might be due to the rapid metabolism and/or excretion of OPEs (Greaves et al., 2016; Poma et al., 2018). It seems reasonable that increased meat and egg consumption were associated with lower urinary metabolite concentrations. Although a small study of toddlers in the USA $(n=41)$ suggested that meat and fish consumption were related to higher DPHP and BDCIPP concentrations in urine, these associations were not statistically significant (Thomas et al., 2017). Moreover, a previous study showed that the OPE levels in bean products were negligible (Ding et al., 2018), thus, an inverse association between high consumption of beans and the urinary BDCIPP concentration is also possible.

Tap water is the most common and cheapest drinking water in China and contains abundant OPEs, particularly halogeno-OPEs, compared to other water sources (e.g., barreled water, bottled water, purified water, and well water) (Ding et al., 2015). A possible hypothesis is that purified water, barreled water, and other drinking water may be associated with lower concentrations of urinary OPE metabolites. Due to the limited number of participants in the other drinking water group, the result that other drinking water was associated with a lower TCEP concentration in urine should be interpreted with caution. However, it is unexpected that barreled water drinking was associated with an increase in urinary BCIPHIPP. Li et al. (2019c) analyzed the concentrations of OPEs in tap water samples from 79 cities in China, in which the contamination of OPEs in tap water from Liuzhou (the sample site of our study) was not severe. In addition, most barreled water-producing manufacturers are regional, and different manufacturing techniques are used according to the enterprise scale and cost. In the sample site of our study, regional barreled water could be a potential source of exposure to OPEs for residents. Considering the limited data on drinking water around the world, more research on different types of drinking water is needed.

\subsection{Limitation and strength}

The main strengths of our study include the large sample size for an exposure study of OPEs and the focus on the vulnerable population of children and adolescents. This is a relatively comprehensive study that examines the relationship between urinary OPE metabolites and dietary factors. The dietary factors include the consumption rate of seven common food groups and the 
sources of daily drinking water. Additionally, the OPE levels in hand wipes were included in the multivariable linear regression models given that dermal contact with dust is commonly considered an important exposure pathway for humans.

Several limitations exist. First, organophosphate diesters could be generated from bio-transformation of OPEs, as well as environmental degradation of parent OPEs and industrial production (Liu et al., 2021). Meanwhile, some of organophosphate diesters are metabolized by various OPEs. For example, DPHP could be generated from TPHP (Su et al., 2014), EHDPP (Shen et al., 2019), and resorcinol bis(diphenylphosphate) (RDP) metabolism (Ballesteros-Gómez et al., 2015). Therefore, the suitability of usage of organophosphate diesters as metabolites of OPEs are needed further assessment. Second, given that other newly discovered OPEs exhibit great detection frequencies and are abundant in multiple environmental matrices (e.g., bis-(2-ethylhexyl)phenyl phosphate (BEHPP), tris(2,4-di-tertbutylphenyl) phosphate (TDTBPP)), these novel OPEs should be monitored in the forthcoming studies. Third, we could not examine long-term exposure to OPEs using a cross-sectional study, and many of OPEs are rapidly metabolized in the human body; thus, a spot urine sample could not be representative of the average body burden of our participants. Fourth, single hand wipe sample could be a potential weakness for OPE measurements, as it is unknown how OPE levels vary in a single person over time. Herein, we measured OPEs in urine and hand wipe samples, whereas the inclusion of other exposure metrics (e.g., passive air samples and indoor dust) may provide additional information on exposure to OPEs. Last, in the present study, food items combined with food frequency questionnaires were used to estimate human dietary intake of OPEs, but this method only provides an approximately estimate of dietary habits during a designed period of sample time (Xu et al., 2017a).

\section{Conclusion}

Our study indicated the widespread exposure to OPEs in children and adolescents. OPE intake via skin wipes could be an important contributor to the total daily of OPEs. Additionally, dietary intake is also a significant exposure source of certain OPEs, particularly vegetable consumption and barreled water drinking. More research is warranted to verify our preliminary findings and evaluate the potential health risks from chronic dietary exposure to OPEs.

\section{Declarations}

\section{Acknowledgments}

The authors would like to thank all collaborators and participants in this project.

\section{Availability of data and materials}

All data generated or analyzed during this study are included in this published article (and its supplementary information).

\section{Funding}

This research was supported by the National Natural Science Foundation of China (No. 42077397; 21577043).

\section{Author contributions}

Project development: Youjie Wang and Surong Mei. Data collection and management: Bingqing Liu, Liqin Hu, and Lulu Song. Sample testing: Meng Yu, Xiang Li, Yaping Li, Ling Liu, and Limei Wang. Data analysis: Meng Yu, Lulu Song, and Liqin Hu. Manuscript writing/editing: Meng Yu and Liqin Hu. Supervision: Surong Mei. All authors read and approved the final manuscript.

\section{Ethics approval and consent to participants}

Ethical approvals were obtained from Tongji Medical College, Huazhong University of Science and Technology. Informed consent was provided by the participant's parents prior to enrollment.

\section{Consent for publication}


Not applicable.

\section{Competing financial interests}

The authors declare no competing financial interests.

\section{References}

1. Abou-Elwafa Abdallah M, Pawar G, Harrad S (2016) Human dermal absorption of chlorinated organophosphate flame retardants; implications for human exposure. Toxicol Appl Pharmacol 291:28-37

2. Ballesteros-Gómez A, Van den Eede N, Covaci A (2015) In vitro human metabolism of the flame retardant resorcinol bis(diphenylphosphate) (RDP). Environ Sci Technol 49:3897-3904

3. Bai XY, Lu SY, Xie L, Zhang B, Song SM, He Y, Ouyang JP, Zhang T (2019) A pilot study of metabolites of organophosphorus flame retardants in paired maternal urine and amniotic fluid samples: potential exposure risks of tributyl phosphate to pregnant women. Environ Sci Process Impacts 21:124-132

4. Bastiaensen M, Ait Bamai Y, Araki A, Goudarzi H, Konno S, Ito S, Miyashita C, Yao Y, Kishi R, Covaci A (2020) Temporal trends and determinants of PFR exposure in the Hokkaido Study. Int J Hyg Environ Health 228:113523

5. Bastiaensen M, Ait Bamai Y, Araki A, Van den Eede N, Kawai T, Tsuboi T, Kishi R, Covaci A (2019) Biomonitoring of organophosphate flame retardants and plasticizers in children: Associations with house dust and housing characteristics in Japan. Environ Res 172:543-551

6. Bastiaensen M, Gys C, Colles A, Verheyen V, Koppen G, Govarts E, Bruckers L, Morrens B, Loots I, De Decker A, Nelen V, Nawrot T, De Henauw S, Van Larebeke N, Schoeters G, Covaci A (2021) Exposure levels, determinants and risk assessment of organophosphate flame retardants and plasticizers in adolescents (14-15 years) from the Flemish Environment and Health Study. Environ Int 147:106368

7. Björnsdotter MK, Romera-García E, Borrull J, de Boer J, Rubio S, Ballesteros-Gómez A (2018) Presence of diphenyl phosphate and aryl-phosphate flame retardants in indoor dust from different microenvironments in Spain and the Netherlands and estimation of human exposure. Environ Int 112:59-67

8. Böckers M, Paul NW, Efferth T (2020) Organophosphate ester tri-o-cresyl phosphate interacts with estrogen receptor a in MCF-7 breast cancer cells promoting cancer growth. Toxicol Appl Pharmacol 395:114977

9. Cao D, Lv K, Gao W, Fu J, Wu J, Fu J, Wang Y, Jiang G (2019) Presence and human exposure assessment of organophosphate flame retardants (OPEs) in indoor dust and air in Beijing, China. Ecotoxicol Environ Saf 169:383-391

10. Cequier E, Sakhi AK, Marcé RM, Becher G, Thomsen C (2015) Human exposure pathways to organophosphate triesters - a biomonitoring study of mother-child pairs. Environ Int 75:159-165

11. Chen Y, Fang J, Ren L, Fan R, Zhang J, Liu G, Zhou L, Chen D, Yu Y, Lu S (2018) Urinary metabolites of organophosphate esters in children in South China: Concentrations, profiles and estimated daily intake. Environ Pollut 235:358-364

12. Chen Y, Jiang L, Lu S, Kang L, Luo X, Liu G, Cui X, Yu Y (2019) Organophosphate ester and phthalate ester metabolites in urine from primiparas in Shenzhen, China: Implications for health risks. Environ Pollut 247:944-952

13. Ding J, Deng T, Xu M, Wang S, Yang F (2018) Residuals of organophosphate esters in foodstuffs and implication for human exposure. Environ Pollut 233:986-991

14. Ding J, Deng T, Ye X, Covaci A, Liu J, Yang F (2019) Urinary metabolites of organophosphate esters and implications for exposure pathways in adolescents from Eastern China. Sci Total Environ 695:133894

15. Ding J, Shen X, Liu W, Covaci A, Yang F (2015) Occurrence and risk assessment of organophosphate esters in drinking water from Eastern China. Sci Total Environ 538:959-965

16. Ding J, Xu Z, Huang W, Feng L, Yang F (2016) Organophosphate ester flame retardants and plasticizers in human placenta in Eastern China. Sci Total Environ 554-555:211-217

17. Greaves AK, Letcher RJ, Chen D, McGoldrick DJ, Gauthier LT, Backus SM (2016) Retrospective analysis of organophosphate flame retardants in herring gull eggs and relation to the aquatic food web in the Laurentian Great Lakes of North America. 
Environ Res 150:255-263

18. Hammel SC, Hoffman K, Phillips AL, Levasseur JL, Lorenzo AM, Webster TF, Stapleton HM (2020) Comparing the Use of Silicone Wristbands, Hand Wipes, And Dust to Evaluate Children's Exposure to Flame Retardants and Plasticizers. Environ Sci Technol 54:4484-4494

19. He C, English K, Baduel C, Thai P, Jagals P, Ware RS, Li Y, Wang X, Sly PD, Mueller JF (2018a) Concentrations of organophosphate flame retardants and plasticizers in urine from young children in Queensland, Australia and associations with environmental and behavioural factors. Environ Res 164:262-270

20. He C, Toms LL, Thai P, Van den Eede N, Wang X, Li Y, Baduel C, Harden FA, Heffernan AL, Hobson P, Covaci A, Mueller JF (2018b) Urinary metabolites of organophosphate esters: Concentrations and age trends in Australian children. Environ Int 111:124-130

21. He C, Wang X (2018) Concentrations of Organophosphate Esters and Their Specific Metabolites in Food in Southeast Queensland, Australia: Is Dietary Exposure an Important Pathway of Organophosphate Esters and Their Metabolites? Environ. Sci Technol 52:12765-12773

22. He C, Wang X, Thai P, Baduel C, Gallen C, Banks A, Bainton P, English K, Mueller JF (2018c) Organophosphate and brominated flame retardants in Australian indoor environments: Levels, sources, and preliminary assessment of human exposure. Environ Pollut 235:670-679

23. Hoffman K, Garantziotis S, Birnbaum LS, Stapleton HM (2015) Monitoring indoor exposure to organophosphate flame retardants: hand wipes and house dust. Environ Health Perspect 123:160-165

24. Hoffman K, Hammel SC, Phillips AL, Lorenzo AM, Chen A, Calafat AM, Ye X, Webster TF, Stapleton HM (2018) Biomarkers of exposure to SVOCs in children and their demographic associations: The TESIE Study. Environ Int 119:26-36

25. Hou M, Shi Y, Na G, Cai Y (2021) A review of organophosphate esters in indoor dust, air, hand wipes and silicone wristbands: Implications for human exposure. Environ Int 146:106261

26. Hou R, Liu C, Gao X, Xu Y, Zha J, Wang Z (2017) Accumulation and distribution of organophosphate flame retardants (PFRs) and their di-alkyl phosphates (DAPs) metabolites in different freshwater fish from locations around Beijing, China. Environ Pollut 229:548-556

27. Hou R, Xu Y, Wang Z (2016) Review of OPFRs in animals and humans: Absorption, bioaccumulation, metabolism, and internal exposure research. Chemosphere 153:78-90

28. Hou M, Shi Y, Na G, Cai Y (2021) A review of organophosphate esters in indoor dust, air, hand wipes and silicone wristbands: Implications for human exposure. Environ Int 146:106261

29. Hu L, Tao Y, Luo D, Feng J, Wang L, Yu M, Li Y, Covaci A, Mei S (2019) Simultaneous biomonitoring of 15 organophosphate flame retardants metabolites in urine samples by solvent induced phase transition extraction coupled with ultraperformance liquid chromatography-tandem mass spectrometry. Chemosphere 233:724-732

30. Huang Y, Tan H, Li L, Yang L, Sun F, Li J, Gong X, Chen D (2020) A broad range of organophosphate tri- and di-esters in house dust from Adelaide, South Australia: Concentrations, compositions, and human exposure risks. Environ Int 142:105872

31. Larsson K, de Wit CA, Sellström U, Sahlström L, Lindh CH, Berglund M (2018) Brominated Flame Retardants and Organophosphate Esters in Preschool Dust and Children's Hand Wipes. Environ Sci Technol 52:4878-4888

32. Li J, Zhao L, Letcher RJ, Zhang Y, Jian K, Zhang J, Su G (2019a) A review on organophosphate Ester (OPE) flame retardants and plasticizers in foodstuffs: Levels, distribution, human dietary exposure, and future directions. Environ Int 127:35-51

33. Li N, Ho W, Wu S, Ying RS, Wang GG, Jones Z, Deng K, W. J (2019b) Organophosphate flame retardants and bisphenol A in children's urine in Hong Kong: has the burden been underestimated? Ecotoxicol. Environ Saf 183:109502

34. Li J, He J, Li Y, Liu Y, Li W, Wu N, Zhang L, Zhang Y, Niu Z (2019c) Assessing the threats of organophosphate esters (flame retardants and plasticizers) to drinking water safety based on USEPA oral reference dose (RfD) and oral cancer slope factor (SFO). Water Res 154:84-93

35. Liu X, Ji K, Choi K (2012) Endocrine disruption potentials of organophosphate flame retardants and related mechanisms in H295R and MVLN cell lines and in zebrafish. Aquat Toxicol 114-115:173-181

Page $17 / 20$ 
36. Liu X, Yu G, Cao Z, Wang B, Huang J, Deng S, Wang Y (2017) Occurrence of organophosphorus flame retardants on skin wipes: Insight into human exposure from dermal absorption. Environ Int 98:113-119

37. Liu Y, Gong S, Ye L, Li J, Liu C, Chen D, Fang M, Letcher RJ, Su G (2021) Organophosphate (OP) diesters and a review of sources, chemical properties, environmental occurrence, adverse effects, and future directions. Environ Int 155:106691

38. Mäkinen MSE, Mäkinen MRA, Koistinen JTB, Pasanen A-L, Pasanen PO, Kalliokoski PJ, Korpi AM (2009) Respiratory and Dermal Exposure to Organophosphorus Flame Retardants and Tetrabromobisphenol A at Five Work Environments. Environ Sci Technol 43:941-947

39. Miller MD, Marty MA, Arcus A, Brown J, Morry D, Sandy M (2002) Differences between children and adults: Implications for risk assessment at California EPA. Int J Toxicol 21:403-418

40. Ospina M, Jayatilaka NK, Wong L-Y, Restrepo P, Calafat AM (2018) Exposure to organophosphate flame retardant chemicals in the U.S. general population: Data from the 2013-2014 National Health and Nutrition Examination Survey. Environ Int 110:32-41

41. Pan W, Zeng D, Ding N, Luo K, Man YB, Zeng L, Zhang Q, Luo J, Kang Y (2020) Percutaneous Penetration and Metabolism of Plasticizers by Skin Cells and Its Implication in Dermal Exposure to Plasticizers by Skin Wipes. Environ Sci Technol 54:10181-10190

42. Pantelaki I, Voutsa D (2020) Occurrence, analysis and risk assessment of organophosphate esters (OPEs) in biota: A review. Mar Pollut Bull 160:111547

43. Percy Z, La Guardia MJ, Xu Y, Hale RC, Dietrich KN, Lanphear BP, Yolton K, Vuong AM, Cecil KM, Braun JM, Xie C, Chen A (2020) Concentrations and loadings of organophosphate and replacement brominated flame retardants in house dust from the home study during the PBDE phase-out. Chemosphere 239:124701

44. Phillips AL, Hammel SC, Hoffman K, Lorenzo AM, Chen A, Webster TF, Stapleton HM (2018) Children's residential exposure to organophosphate ester flame retardants and plasticizers: Investigating exposure pathways in the TESIE study. Environ Int 116:176-185

45. Poma G, Sales C, Bruyland B, Christia C, Goscinny S, Van Loco J, Covaci A (2018) Occurrence of Organophosphorus Flame Retardants and Plasticizers (PFRs) in Belgian Foodstuffs and Estimation of the Dietary Exposure of the Adult Population. Environ Sci Technol 52:2331-2338

46. Poma G, Yin S, Tang B, Fujii Y, Cuykx M, Covaci A (2019) Occurrence of Selected Organic Contaminants in Edible Insects and Assessment of Their Chemical Safety. Environ Health Perspect 127:127009

47. Rock KD, Armour St, Horman G, Phillips B, Ruis A, Stewart M, Jima AK, Muddiman D, Stapleton DC, Patisaul HM, H. B (2020) Effects of Prenatal Exposure to a Mixture of Organophosphate Flame Retardants on Placental Gene Expression and Serotonergic Innervation in the Fetal Rat Brain. Toxicol Sci 176:203-223

48. Scheuplein R, Charnley G, Dourson M (2002) Differential sensitivity of children and adults to chemical toxicity. I. Biological basis. Regul Toxicol Pharmacol 35:429-447

49. Shen J, Zhang Y, Yu N, Crump D, Li J, Su H, Letcher RJ, Su G (2019) Organophosphate Ester, 2-Ethylhexyl Diphenyl Phosphate (EHDPP), Elicits Cytotoxic and Transcriptomic Effects in Chicken Embryonic Hepatocytes and Its Biotransformation Profile Compared to Humans. Environ Sci Technol 53:2151-2160

50. Stapleton HM, Misenheimer J, Hoffman K, Webster TF (2014) Flame retardant associations between children's handwipes and house dust. Chemosphere 116:54-60

51. Su G, Crump D, Letcher RJ, Kennedy SW (2014) Rapid in vitro metabolism of the flame retardant triphenyl phosphate and effects on cytotoxicity and mRNA expression in chicken embryonic hepatocytes. Environ Sci Technol 48:13511-13519

52. Sugeng EJ, Leonards PEG, van de Bor M (2017) Brominated and organophosphorus flame retardants in body wipes and house dust, and an estimation of house dust hand-loadings in Dutch toddlers. Environ Res 158:789-797

53. Tan H, Chen D, Peng C, Liu X, Wu Y, Li X, Du R, Wang B, Guo Y, Zeng EY (2018) Novel and Traditional Organophosphate Esters in House Dust from South China: Association with Hand Wipes and Exposure Estimation. Environ Sci Technol 52:11017-11026 
54. Tan H, Yang L, Yu Y, Guan Q, Liu X, Li L, Chen D (2019) Co-Existence of Organophosphate Di- and Tri-Esters in House Dust from South China and Midwestern United States: Implications for Human Exposure. Environ Sci Technol 53:4784-4793

55. Tao Y, Shang Y, Li J, Feng J, He Z, Covaci A, Wang P, Luo J, Mao X, Shi B, Hu L, Luo D, Mei S (2018) Exposure to organophosphate flame retardants of hotel room attendants in Wuhan City. China Environ Pollut 236:626-633

56. Tao Y, Hu L, Liu L, Yu M, Li Y, Li X, Liu W, Luo D, Covaci A, Xia W, Xu S, Li Y, Mei S (2021) Prenatal exposure to organophosphate esters and neonatal thyroid-stimulating hormone levels: A birth cohort study in Wuhan. China Environ Int 156:106640

57. Tay JH, Sellström U, Papadopoulou E, Padilla-Sánchez JA, Haug LS, de Wit CA (2018) Assessment of dermal exposure to halogenated flame retardants: Comparison using direct measurements from hand wipes with an indirect estimation from settled dust concentrations. Environ Int 115:285-294

58. Thomas MB, Stapleton HM, Dills RL, Violette HD, Christakis DA, Sathyanarayana S (2017) Demographic and dietary risk factors in relation to urinary metabolites of organophosphate flame retardants in toddlers. Chemosphere 185:918-925

59. Tulve NS, Suggs JC, McCurdy T, Hubal C, Moya EA, J (2002) Frequency of mouthing behavior in young children. J Expo Sci Environ Epidemiol 12:259-264

60. USEPA (2011) Exposure Factors Handbook; EPA/600/R-09/052F. U.S. Environmental Protection Agency, Washington, DC

61. Van den Eede N, Heffernan AL, Aylward LL, Hobson P, Neels H, Mueller JF, Covaci A (2015) Age as a determinant of phosphate flame retardant exposure of the Australian population and identification of novel urinary PFR metabolites. Environ Int 74:1-8

62. Van den Eede N, Maho W, Erratico C, Neels H, Covaci A (2013) First insights in the metabolism of phosphate flame retardants and plasticizers using human liver fractions. Toxicol Lett 223:9-15

63. Wang X, Zhu Q, Yan X, Wang Y, Liao C, Jiang G (2020) A review of organophosphate flame retardants and plasticizers in the environment: Analysis, occurrence and risk assessment. Sci Total Environ 731:139071

64. Wei GL, Li DQ, Zhuo MN, Liao YS, Xie ZY, Guo TL, Li JJ, Zhang SY, Liang ZQ (2015) Organophosphorus flame retardants and plasticizers: sources, occurrence, toxicity and human exposure. Environ Pollut 196:29-46

65. Xu F, Giovanoulis G, van Waes S, Padilla-Sanchez JA, Papadopoulou E, Magnér J, Haug LS, Neels H, Covaci A (2016) Comprehensive Study of Human External Exposure to Organophosphate Flame Retardants via Air, Dust, and Hand Wipes: The Importance of Sampling and Assessment Strategy. Environ Sci Technol 50:7752-7760

66. Xu F, Tay JH, Covaci A, Padilla-Sánchez JA, Papadopoulou E, Haug LS, Neels H, Sellström U, de Wit CA (2017a) Assessment of dietary exposure to organohalogen contaminants, legacy and emerging flame retardants in a Norwegian cohort. Environ Int 102:236-243

67. Xu Q, Wu D, Dang Y, Yu L, Liu C, Wang J (2017b) Reproduction impairment and endocrine disruption in adult zebrafish (Danio rerio) after waterborne exposure to TBOEP. Aquat Toxicol 182:163-171

68. Yang C, Harris SA, Jantunen LM, Siddique S, Kubwabo C, Tsirlin D, Latifovic L, Fraser B, St-Jean M, De La Campa R, You H, Kulka R, Diamond ML (2019) Are cell phones an indicator of personal exposure to organophosphate flame retardants and plasticizers? Environ. Int 122:104-116

69. Ye L, Meng W, Huang J, Li J, Su G (2021) Establishment of a Target, Suspect, and Functional Group-Dependent Screening Strategy for Organophosphate Esters (OPEs): "Into the Unknown" of OPEs in the Sediment of Taihu Lake, China. Environ Sci Technol 55:5836-5847

70. Zhang X, Zou W, Mu L, Chen Y, Ren C, Hu X, Zhou Q (2016) Rice ingestion is a major pathway for human exposure to organophosphate flame retardants (OPFRs) in China. J Hazard Mater 318:686-693

71. Zhang B, Lu S, Huang M, Zhou M, Zhou Z, Zheng H, Jiang Y, Bai X, Zhang T (2018a) Urinary metabolites of organophosphate flame retardants in 0-5-year-old children: Potential exposure risk for inpatients and home-stay infants. Environ Pollut 243:318-325

72. Zhang T, Bai XY, Lu SY, Zhang B, Xie L, Zheng HC, Jiang YC, Zhou MZ, Zhou ZQ, Song SM, He Y, Gui MW, Ouyang JP, Huang HB, Kannan K (2018b) Urinary metabolites of organophosphate flame retardants in China: Health risk from tris(2chloroethyl) phosphate (TCEP) exposure. Environ Int 121:1363-1371 
73. Zhao L, Zhang Y, Deng Y, Jian K, Li J, Ya M, Su G (2020) Traditional and emerging organophosphate esters (OPEs) in indoor dust of Nanjing, eastern China: Occurrence, human exposure, and risk assessment. Sci Total Environ 712:136494

\section{Figures}
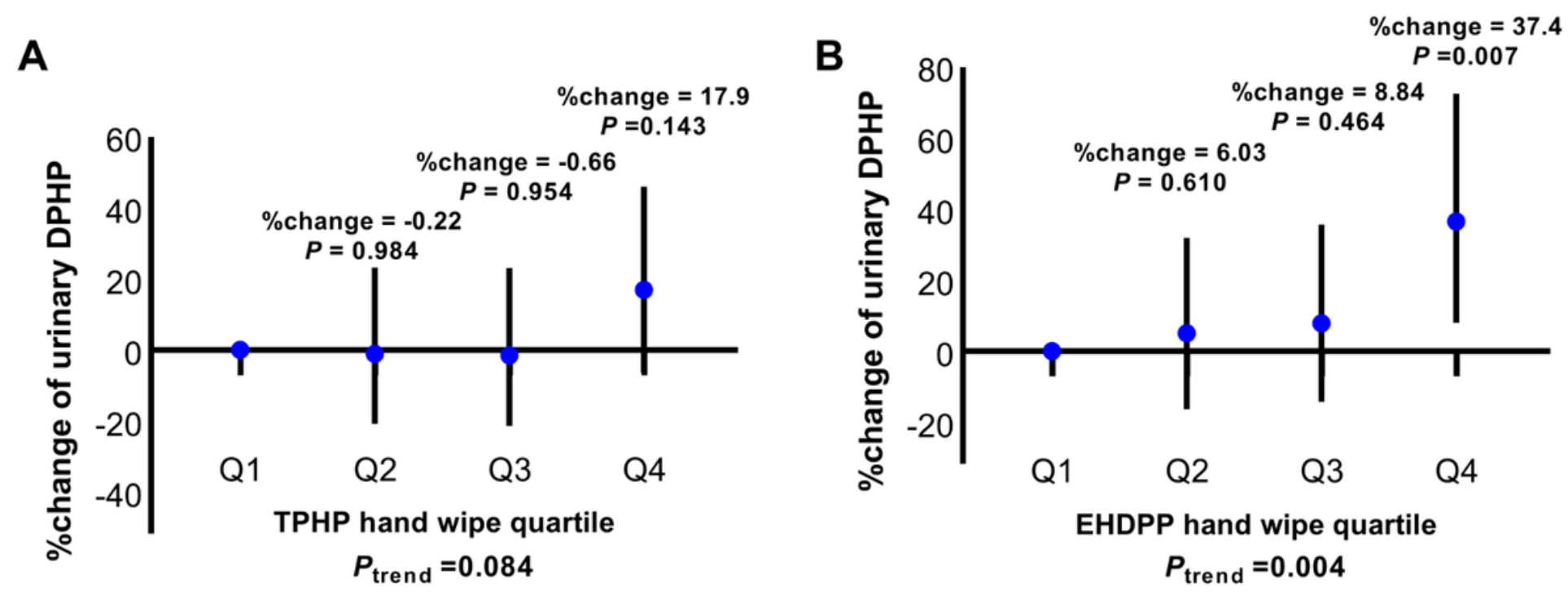

Figure 1

Adjusted linear regression associations between TPHP (A) and EHDPP (B) levels on hand wipes and their urinary metabolite DPHP. Models are adjusted for sex and age, maternal education, and all food groups.

\section{Supplementary Files}

This is a list of supplementary files associated with this preprint. Click to download.

- Supplementarymaterials.docx 